\title{
INTEGRALS ALLIED TO AIRY'S INTEGRALS
}

\section{by T. M. MACROBERT}

(Received 13th February, 1956)

1. Introductory. Airy's integrals

$$
\int_{0}^{\infty} \cos \left(\lambda^{3} \pm x \lambda\right) d \lambda
$$

can be expressed $([1],[2])$ in terms of Bessel functions. In this paper integrals of the types

$$
\int_{0}^{\infty} \cos \sin \left(\lambda^{n} \pm x \lambda^{l}\right) \lambda^{k-1} d \lambda
$$

are discussed. Various subsidiary formulae are given in $\S 2$, some integrals of the type

$$
\int_{0}^{\infty} \exp \left(-\lambda^{n}\right) f\left(\lambda^{l}\right) \lambda^{k-1} d \lambda
$$

are evaluated in $\S 3$, and from these the integrals of the Airy type are derived in $\S 4$.

2. Formulae required in the proof. The first of these is the Gamma function formula

$$
\Gamma(z) \Gamma\left(z+\frac{1}{m}\right) \ldots \Gamma\left(z+\frac{m-1}{m}\right)=(2 \pi)^{1 m-1} m-m z \Gamma(m z) .
$$

The second is Ragab's formula ([2], p. 406, ex. 27, [3])

$$
K_{\mu}(z) K_{\nu}(z)=\frac{1}{4 z \sqrt{\pi}} \sum_{i,-i} \frac{1}{i} E\left(\frac{1+\mu+\nu}{2}, \frac{1+\mu-\nu}{2}, \frac{1-\mu+\nu}{2}, \frac{1-\mu-\nu}{2}: \frac{1}{2}: e^{i \pi} z^{2}\right) .
$$

The following two formulae are also required.

If $m$ is a positive integer and if $R(k)>0,([2], \mathrm{p} .406, \mathrm{ex} .30)$,

$$
\int_{0}^{\infty} e^{-\lambda} \lambda^{k-1} E\left(p ; \alpha_{r}: q ; \rho_{s}: z / \lambda^{m}\right) d \lambda=m^{k-\frac{1}{2}}(2 \pi)^{\frac{k-1}{m}} E\left(p+m ; \alpha_{r}: q ; \rho_{s}: z / m^{m}\right)
$$

where $\alpha_{p+\nu+1}=(k+\nu) / m, \nu=0,1,2, \ldots, m-1$.

If $m$ is a positive integer and if $|\operatorname{amp} z|<\pi,([2]$, p. 407, ex. 32),

$$
\frac{1}{2 \pi i} \int e^{\zeta \zeta-\rho} E\left(p ; \alpha_{r}: q ; \rho_{s}: \zeta^{m} z\right) d \zeta=m^{t-\rho}(2 \pi)^{\mathbf{t}^{m-1}} E\left(p ; \alpha_{r}: q+m ; \rho_{s}: z m^{m}\right),
$$

where the contour starts at $-\infty$ on the real axis, passes round the origin in the positive direction, and returns to $-\infty$, and $\rho_{a+\nu+1}=(\rho+\nu) / m, \nu=0,1,2, \ldots, m-1$.

3. Some infinite integrals. Consider the integral

$$
\int_{0}^{\infty} \exp \left(-\lambda^{n}+z \lambda^{l}\right) \lambda^{k-1} d \lambda
$$

where $n$ and $l$ are positive integers such that $l<n$, and $R(k)>0$. On expanding $\exp \left(z \lambda^{l}\right)$ in powers of $z$ and putting $\lambda=\mu^{1 / n}$, this becomes

$$
\frac{1}{n} \sum_{r=0}^{\infty} \frac{z^{r}}{r !} \Gamma\left(\frac{k+r l}{n}\right)
$$

and therefore 


$$
\begin{aligned}
\int_{0}^{\infty} \exp \left(-\lambda^{n}+z \lambda^{l}\right) \lambda^{k-1} d \lambda \\
=\frac{1}{n} \sum_{t=0}^{n-1} \Gamma\left(\frac{k+t l}{n}\right) \frac{z^{t}}{t !} F\left\{\begin{array}{c}
\frac{k+t l}{n l}, \frac{k+t l+n}{n l}, \ldots, \frac{k+t l+(l-1) n}{n l}: l^{l}\left(\frac{z}{n}\right)^{n} \\
\frac{t+1}{n}, \frac{t+2}{n}, \ldots * \ldots, \frac{t+n}{n}
\end{array}\right\},
\end{aligned}
$$

the asterisk indicating that the parameter $n / n$ is omitted.

Now assume that $n$ is odd, replace $z$ by $-1 / z$, and apply $(1)$ with $(k+t l) /(n l)$ for $z$ and $l$ for $m$ and also with $(t+1) / n$ for $z$ and $n$ for $m$; then the equation can be written

$$
\begin{aligned}
& \int_{0}^{\infty} \exp \left(-\lambda^{n}\right) E\left(:: z / \lambda^{l}\right) \lambda^{k-1} d \lambda \\
= & n^{-3 / 2 l-t+k / n}(2 \pi)^{-n-t l} \sum_{t=0}^{n-1}\left(-n l^{-l / n} z\right)^{-t} E\left\{\begin{array}{c}
\frac{k+t l}{n l}, \frac{k+t l+n}{n l}, \ldots, \frac{k+t l+(l-1) n}{n l}: l^{-l}(n z)^{n} \\
\frac{t+1}{n}, \frac{t+2}{n}, \ldots * \ldots, \frac{t+n}{n}
\end{array}\right\} .
\end{aligned}
$$

On generalising, using (3) and (4), it is found that, if $n$ and $l$ are positive integers such that $n$ is odd and $l<n$, and if $R(k)>0$,

$$
\begin{aligned}
& \exp \left(-\lambda^{n}\right) E\left(p ; \alpha_{r}: q ; \rho_{s}: z / \lambda^{l}\right) \lambda^{k-1} d \lambda \\
& =n^{\Sigma \alpha_{r}-\Sigma \rho_{s}-\frac{1}{2} p+\frac{1}{2} q-3 / 2} l^{-\frac{1}{2}+k / n}(2 \pi)^{\left(t-\frac{1}{2} n\right)(p-q)+\frac{1}{2} n-1 l} \\
& \times \sum_{l=0}^{n-1}\left(-l^{-l / n} n^{q-p+1} z\right)^{-t} E\left\{\begin{array}{l}
\frac{k+t l}{n l}, \ldots, \frac{k+t l+(l-1) n}{n l}, \frac{\alpha_{1}+t}{n}, \ldots, \frac{\alpha_{p}+t+n-1}{n}: l^{-l}\left(n^{q-p+1} z\right)^{n} \\
\frac{t+1}{n}, \ldots * \ldots, \frac{t+n}{n}, \frac{\rho_{1}+t}{n}, \ldots, \frac{\rho_{q}+t+n-1}{n}
\end{array}\right\} .
\end{aligned}
$$

Note. If $n$ is even the argument of the $E$-function should be multiplied by $e^{ \pm i \pi}$.

For example, on applying formula (2) it is found that

$$
\left.\begin{array}{l}
\int_{0}^{\infty} \exp \left(-\lambda^{n}\right) K_{\mu}\left(z / \lambda^{l}\right) K_{v}\left(z / \lambda^{l}\right) \lambda^{k-1} d \lambda \\
=(2 \sqrt{ } 2 z)^{-1} n^{-3 / 2}(2 l)^{-1+(k+l) / n}(2 \pi)^{1-n-l} \sum_{i,-i} \frac{1}{i} \sum_{t=0}^{n-1}\left\{(2 l)^{-2 l / n} n^{-2} z^{2}\right\}^{-t} \\
\quad \times E\left\{\begin{array}{l}
\frac{k+l+2 t l}{2 n l}, \ldots, \frac{k+l+2 t l+(2 l-1) n}{2 n l}, \frac{1+\mu+\nu+2 t}{2 n}, \ldots, \\
\frac{1-\mu-\nu+2 t+2 n-2}{2 n}: \frac{e^{i n \pi}(z / n)^{2 n}}{(2 l)^{2 l}}
\end{array}\right\}, \\
\frac{t+1}{n}, \ldots * \ldots, \frac{t+n}{n}, \frac{1+2 t}{2 n}, \ldots, \frac{1+2 t+2 n-2}{2 n}
\end{array}\right\},
$$

where $n$ and $l$ are positive integers such that $n$ is odd and $n>2 l$, and $R(k)>-l$.

4. Integrals of the Airy type. In formula (5) swing the line of integration through a positive angle $\pi /(2 n)$, so that $\lambda$ becomes $\eta e^{i \pi /(2 n)}$, and let $z= \pm x e^{i(n-l) \pi /(2 n)}$, where $x$ is real and positive ; then 


$$
\begin{aligned}
\int_{0}^{\infty} \exp ( & \left.-i \eta^{n} \pm i x \eta^{l}\right) \eta^{k-1} d \eta=\frac{1}{n} e^{-i k \pi /(2 n)} \sum_{t=0}^{n-1} \Gamma\left(\frac{k+t l}{n}\right) \frac{( \pm x)^{t}}{t !} \\
\times e^{i t(n-l) \pi /(2 n)} F & \left\{\begin{array}{l}
\left.\frac{k+t l}{n l}, \frac{k+t l+n}{n l}, \ldots, \frac{k+t l+(l-1) n}{n l} ; e^{i(n-l) \pi / 2} l l\left(\frac{ \pm x}{n}\right)^{n}\right\} \\
\frac{t+1}{n}, \frac{t+2}{n}, \ldots * \ldots, \frac{t+n}{n}
\end{array}\right\}, \ldots \ldots(9)
\end{aligned}
$$

where $n>R(k)>0$ and $n>l$.

On putting $n=3, l=1, k=1$, and equating real parts, Airy's integrals are obtained.

The same method may be applied for other values of $n$ and $l$.

For instance, if $n=5, l=3$, and if $k$ is real and such that $0<k<5, x$ real and positive,

$$
\begin{aligned}
\int_{0}^{\infty} \sin \left(\eta^{5} \pm x \eta^{3}\right) \eta^{k-1} d \eta=\frac{1}{5} \sum_{t=0}^{4} & \Gamma\left(\frac{k+3 t}{5}\right) \cos \left(\frac{k-2 t}{10} \pi\right) \frac{(\mp x)^{t}}{t !} \\
\times F & \left\{\begin{array}{l}
\frac{k+3 t}{15}, \frac{k+3 t+5}{15}, \frac{k+3 t+10}{15} ; \pm 27\left(\frac{x}{5}\right)^{5} \\
\frac{t+1}{5}, \frac{t+2}{5}, \ldots * \ldots, \frac{t+5}{5}
\end{array}\right\} .
\end{aligned}
$$

Note. From these formulae numerous others can be deduced. For instance, if the cosines with arguments $\pm x$ on the left of (10) are added, integrals of the type

$$
\int_{0}^{\infty} \cos \eta^{5} \cos \left(x \eta^{3}\right) \eta^{k-1} d \eta
$$

are obtained. The second cosine and the functions on the right can then be expressed as $E$-functions and generalised.

\section{REFERENCES} $188-190$.

1. Watson, G. N., A treatise on the theory of Bessel functions (2nd edition, Cambridge, 1944),

2. MacRobert, T. M., Functions of a complex variable (4th edition, London, 1954), 402-403.

3. Ragab, F. M. A product of two $E$-functions expressed as a sum of two $E$-functions, Proc. Glasgow Math. Assoc. 2 (1955), 125.

\section{The Untversity}

Glasgow 